\title{
Research Paper: Role of Emotion Regulation in Psychopathology
}

\author{
Alireza Azizi' ${ }^{1}$ Parvaneh Mohammadkhani ${ }^{2 *}$, Abbas Pourshahbaz ${ }^{2}$, Behrouz Doulatshahi², Saba Moghaddam ${ }^{3}$ \\ 1. Department of Psychiatry, School of Medicine, Babol University of Medical Sciences, Babol, Iran. \\ 2. Department of Clinical Psychology, University of Social Welfare \& Rehabilitation Sciences, Tehran, Iran. \\ 3. Department of Psychology, Faculty of Psychology \& Education, Allameh Tabataba'i University, Tehran, Iran.
}

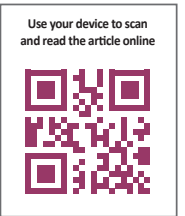

ditation: Azizi A, Mohammadkhani P, Pourshahbaz A, Doulatshahi B, Moghaddam S. Role of Emotion Regulation in Psychopathology. Iranian Rehabilitation Journal. 2018; 16(2):113-120. https://doi.org/10.32598/IRJ.16.2.113

dol: : https://doi.org/10.32598/IRJ.16.2.113

Article info:

Received: 25 Dec 2017

Accepted: 10 Mar 2018

\section{Keywords:}

Psychopathology, Emotion regulation, Skills, Mediation

\section{A B S T RACT}

Objectives: The associations between emotional skills encompassed in emotion regulation model and psychopathology were examined by investigating its ability to modify the emotionmediated association between other emotion regulation skills and psychopathology.

Methods: A total of 465 students were selected using proposal sampling method. Data were collected using Emotion Regulation Skill Questionnaire (ERSQ) and Brief Symptom Inventory (BSI). Data were analyzed using bootstrapping-enhanced mediation analysis that considered the ability to modify emotions as a mediator, psychopathology as dependent, and other emotional skill as independent and Pearson's correlation coefficient.

Results: A significant negative correlation was established between emotional skills and psychopathology in a range from 0.13 (bodily perception of sensations) to 0.31 (modification) ( $\rho=-0.31, P<0.01$ ). The total effect for the variables of clarity, understanding, acceptance and tolerance, confrontation, self-support, attention, and sensation was significant at $\mathrm{P}<0.05$, thereby rendering the mediation analysis plausible. The indirect effect of all variables was also significant. Therefore, all the variables have partial mediating effects. The direct effect of the variable of attention, sensation, and understanding on the psychological disturbances was not significant $(\mathrm{P}>0.05)$, and modification between these three skills and psychopathology exerted a complete mediating effect. The direct impact of emotional clarity, acceptance and tolerance, confrontation, and self-support was significant. Thus, emotion modification between the skills of emotional clarity, acceptance and tolerance, confrontation, and self-support with psychological disturbance had partial mediating effects.

Discussion: The ability to modify emotions is the pathway through which other emotional skills might exert their influence on the mental health.

\section{* Corresponding Author:}




\section{Introduction}

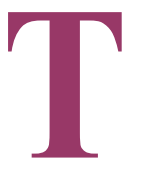

he classification of psychopathology dates back to the mid- $19^{\text {th }}$ century when there was an urgent need for information associated with the prevalence of mental disorders [1]. Such efforts are evident in the first and subsequent editions of the Diagnostic and Statistical Manual of Mental Disorders (DSM). Subsequently, different editions of the DSM were used for categorizing the mental disorders into separate and defined groups that highly overlapped. For instance, the diagnostic criteria for Generalized Anxiety Disorder (GAD) and Major Depressive Disorder (MDD) overlapped each other with respect to four symptoms [2].

Accordingly, transdiagnostic approaches were established in order to identify the transdiagnostic factors. In previous studies, the positive and negative effect [3], neuroticism [4], repetitive negative thinking [5], and emotion regulation $[6,7]$ have been cited as transdiagnostic factors. Moreover, the National Institute of Mental Health (NIMH) developed Research Domain Criteria (RDoC) supporting the transdiagnostic theory. The criteria provided a framework for the classification of the mechanisms underlying psychopathology into five general and broad domains including positive rating systems, negative rating systems, cognitive systems, systems for social processes, as well as, arousal and regulatory systems. As a whole, the process of emotion regulation cannot be considered as a simple collection of $\mathrm{RDoC}$ domains, rather it refers to a unique regulatory process that is conceptualized as the sixth domain within the $\mathrm{RDoC}$ matrix; thus, emotion regulation is the functional consequence of these five domains and recognized as a process variable in the transdiagnostic approach [8].

Thompson (1994) defined emotion regulation as "the extrinsic and intrinsic processes responsible for monitoring, evaluating, and modifying emotional reactions, especially their intensive and temporal features, to accomplish one's goals" [9]. Notably, that emotion regulation deficits have high comorbidity with the symptoms of psychopathology $[10,11]$. Similarly, the results of longitudinal studies have shown that such deficits are not only considered as the symptoms but also recognized as the antecedents of mental disorders $[12,13]$. Therefore, the model of Adaptive Coping with Emotions (ACE) was developed on the basis of emotion regulation deficits in various disorders as well as the interaction of such skills with mental health [14].
The ACE-based adaptive emotion regulation is considered as a situation-dependent interaction between a set of skills, including the ability to (a) be consciously aware of emotions, (b) identify and label emotions, (c) identify what causes and maintains the emotions, (d) modify the emotions in an adaptive manner, (e) accept and tolerate emotions, (f) approach and confront with situations triggering negative emotions, and $(\mathrm{g})$ provide effective self-support in distressing situations [15]. Berking et al. (2010) also developed an Affect Regulation Training (ART) based on the ACE model. Notably, the ART is a transdiagnostic treatment program targeting emotion regulation deficits that can be added to common treatments in order to improve the individual's mental health [16].

Moreover, the findings of cross-sectional studies have suggested a significant relationship between the collection of skills within the ACE model and mental health in nonclinical samples [17-19]. Also, such skills have been less applicable in clinical as compared to non-clinical samples [17-19]. The improvement of the use of skills within this model during cognitive-behavioral therapy has been similarly associated with a decrease in the mental symptoms [18]. Nevertheless, the results of a study on inpatient clinical samples demonstrated that only the ability to modify, accept, and tolerate negative emotions play a significant role in identifying patients' conditions (as compared to a control group selected from the society), predicting the severity of psychopathological symptoms at the pre-treatment stage, as well as, forecasting the changes in the symptoms [17]. Moreover, the significant predictor of relapse during and after the treatment of alcohol dependence was reported as the ability to tolerate negative emotions [12].

Therefore, the skills of acceptance, tolerance, and especially modification in the model developed by Berking et al. (2010) are of utmost importance. The ability to modify negative emotions can prevent the chronic and severe negative emotions, as well as, reduce the intensity and continuity of chronic negative emotions without the use of impulsive strategies [19]. Conversely, the attempts to modify emotions are unsuccessful occasionally, thereby necessitating the acceptance-based skills [20]. Accordingly, Berking et al. (2012) concluded that the ability to modify negative emotions can fully mediate the relationship between the symptoms and skills of the ACE model except for acceptance and tolerance of emotions. Thus, it seems that the ability to modify, tolerate, and acceptance emotions is a pathway through which emotion regulation skills can impact the mental health.

In addition, the cultural values can greatly influence the emotion regulation strategies [21] and processes. For 
instance, studies about differences in regulating emotions have focused on cultural differences in the use of emotion suppression strategy. This strategy consists of external restraint of the expression of emotions. Various studies have demonstrated that Asian population (such as Hong Kong, Japan, and Asian Americans) are more likely to suppress their emotions than Europeans [22-24]. Therefore, culture could also determine the adaptability of emotion regulation (whether this emotion regulation strategy or skill in an individual's psychological wellbeing is good or bad). Alternatively, the emotion regulation is adaptive when it is in agreement with the cultural context and maladaptive when not in accordance with the cultural context $[25,26]$. The emotional regulation skills are highly cultural-dependent, and the relationship between these skills has not yet been studied in Iran.

Considering that this model has been approved in the German culture and similar studies have not yet been conducted in Iran and Asian culture, it is essential to investigate the mediating role of regulation skills and the mechanism of the effect of deficits in emotion regulation skills on psychopathology in Iranian culture. Thus, the present study aimed to determine the role of emotion regulation skills, especially modification in psychopathology.

\section{Methods}

The study participants included 465 University students in an age range of 18-45 (mean=25.11, standard deviation=4.13) years. Tehran University of Medical Sciences, University of Social Welfare and Rehabilitation Sciences, and University of Tehran were selected by cluster sampling. Since the model consisted of 29 free parameters, the ideal sample size was estimated at 580 individuals. A total of 600 questionnaires were distributed, of which, 465 were returned.

The sampling was conducted between June and September 2016. The participants signed the consent forms. The willingness and unwillingness to participate in this study was considered as the main inclusion criterion. Instructions associated with human research were also provided to the participants. To compensate for the efforts and encourage the individuals, they were informed about obtaining feedback regarding their emotion regulation skills and mental health status in the future, and the results of the present study would also be at their disposal.

\section{Assessment tools}

Emotion Regulation Skills Questionnaire (ERSQ): The questionnaire included 27 items assessing the emotion regulation skills over the past week on a 5-point Likert-type scale (from "never" to "almost always"). The questionnaire also contained nine sub-scales including attention to feelings, bodily perception of sensations, understanding of feeling, acceptance of feeling, tolerance of feeling, readiness to confront unwanted emotions, self-support, as well as, modification. For the overall scale, the internal consistency was 0.93 for the trait and 0.90 for the scales of the state version. The test-retest reliability for the subscales was between 0.58 and 0.76 for the trait version and between 0.48 and 0.74 for the state version. [18]. According to the theoretical expectations, a negative and significant correlation was reported between the ERSQ-awareness and Difficulties in Emotion Eegulation Scale (DERS)-lack of awareness $(\mathrm{r}=-0.69)$, the ERSQ-clarity and DERS-limited clarity $(\mathrm{r}=-0.68)$, ERSQ-acceptance/tolerance and DERS-lack of acceptance $(\mathrm{r}=-0.52)$, and ERSQ-modification and DERS-limited access to emotion regulation strategies $(\mathrm{r}=-0.70)$. Furthermore, ERSQ-acceptance and tolerance is negatively and significantly correlated with the subscale of suppression in Meta-cognitive Regulation Scale (MERS) ( $\mathrm{r}=-0.59)$; also, a negative correlation was established between the ERSQ-modification and the expectancy of successfully regulating the negative affect subscale of negative mood regulation scale (NMR) $(\mathrm{r}=-0.71)$ [19]. In Iranian population Cronbach's Alpha of the Persian version was 0.93 . ERSQ score revealed a high negative correlation with each of the 4 factors of the GHQ and high positive correlation with DERS [27].

Brief Symptoms Inventory (BSI) is employed as a screening tool for diagnosing the clinical disorders. This self-report tool included 53 statements, whose scored were based on a 5-point Likert-type scale (from 0 meaning "never" to 4 indicating "very high"). BSI consists of 9 sub-scales including depression, anxiety, anger-hostility, somatization, obsessive-compulsive, interpersonal sensitivity, phobic anxiety, paranoid ideation, and psychoticism [28]. Dobson and Mohammadkhani (2007a) reported the high correlation between BSI-depression subscale and BDI-II in an Iranian study sample ( $\mathrm{r}=0.87)$ [29]. The 9-factor model in the Iranian society was a better fit as compared to the single-factor. Cronbach's alpha for the total scale of the items has been estimated as 0.96 in a range from $0.71-0.87$ for subscales [30].

\section{Statistical analysis}

The mediating effects were tested via the structural equation analysis using AMOS V. 21 software. To evaluate the mediator role of a variable, the dependent variable must be regressed on the independent and mediator 
variables. Also, the mediator is regressed on the independent variable. Significant indirect effect confirms the existence of partial mediation. Moreover, if the direct effect of the independent variable on the dependent variable is significant after controlling the indirect effect, the mediating impact is complete. In the approaches developed by Baron and Kenny (1986) [31] and Sobel (1982) [32], such effects were estimated in separate regression analyses. In this regard, the structural equation analysis is superior to other classical approaches as all the direct and indirect effects have been embedded in a model, and the possibility to estimate the confidence intervals is also provided. The asymmetric confidence intervals are calculated similarly via bootstrapping methods. Notably, such an effect is significant, and no zero is detected within the confidence intervals of bootstrapping [19]. In the present study, the ability to modify emotions was considered as mediator, psychopathology as dependent, and other emotional skill as independent variables.

\section{Results}

The descriptive data obtained from this study were presented in Table 1. Kurtosis and skewness in none of the variables were $>1$ and -1 , indicating that the normal distribution of the data.

Table 2 illustrated the Pearson's correlation coefficient between emotion regulation skills and psychopathology. A negative and significant correlation was established between skills of attention to feelings ( $\rho=-0.16, P<0.01$ ), emotional clarity $(\rho=-0.26, P<0.01)$, bodily perception of sensations $(\rho=-0.13, P<0.05)$, understanding of emotions $(\rho=-0.17, \mathrm{P}<0.01)$, acceptance and tolerance $(\rho=-0.29, P<0.01)$, readiness to confrontation $(\rho=-0.30$, $\mathrm{P}<0.01)$, self-support $(\rho=-0.28, \mathrm{P}<0.01)$, modification ( $\rho=-0.31, P<0.01$ ), and the total score of emotion regulation skills and mental disturbances. Thus, the mediating role of emotional regulation skills can be examined.

According to Table 3, the total effect for the variables of clarity, understanding, acceptance and tolerance, confrontation, and self-support was significant at $\mathrm{P}<0.01$ and the total effect for attention and sensation was significant at $\mathrm{P}<0.05$; therefore, the mediation analysis is plausible. The indirect effect of all variables except for confrontation was also significant at $\mathrm{P}<0.01$. Also, the indirect effect of confrontation at $\mathrm{P}<0.05$ was reported to be significant. Therefore, all the variables have partial mediating effects. Finally, the direct effect of the variables of attention, sensation, and understanding on psychological disturbances was not found to be significant $(\mathrm{P}>0.05)$, and modification between these three skills and psychopathology exhibited a complete mediating effect. The direct impact of emotional clarity, acceptance and tolerance, confrontation, and self-support were also significant at $\mathrm{P}<0.05$; however, only the direct effect of support was significant at $\mathrm{P}<0.01$. Thus, emotion modification among the skills of emotional clarity, acceptance and tolerance, confrontation, and self-support with psychological disturbance had partial mediating effects.

\section{Discussion}

The above results indicated that all emotion regulation skills are inversely correlated with psychological distur-

Table 1. Means, standard deviations, kurtosis, and skewness of ERSQ scales and BSI

\begin{tabular}{cccccc}
\hline Variables & Mean & SD & Kurtosis & Skewness \\
\hline Awareness & 2.69 & 0.73 & -0.39 & 0.36 \\
Sensations & 2.53 & 0.86 & -0.49 & -0.04 \\
Clarity & 2.85 & 0.74 & -0.37 & -0.63 & 0.13 \\
Understanding & 2.55 & 0.86 & -0.64 & 0.40 \\
Acceptance/tolerance & 2.86 & 0.67 & -0.66 & -0.42 \\
Readiness to confront & 2.66 & 0.79 & -0.41 & -0.53 & 0.01 \\
Self-support & 2.53 & 0.82 & 0.84 & -0.45 & -0.15 \\
Modification & 2.64 & 0.47 & -0.36 & 0.20 \\
ERSQ-total & 2.68 & 0.58 & 0.53 & -0.13 \\
\hline BSI-total & 1.03 & & & 0.13 \\
\hline
\end{tabular}


Table 2. Bivariate correlations (Pearson) between ERSQ subscales and indicator of psychopathology

\begin{tabular}{|c|c|c|c|c|c|c|c|c|c|c|}
\hline Variables & 1 & 2 & 3 & 4 & 5 & 6 & 7 & 8 & 9 & 10 \\
\hline 1. Awareness & 1 & & & & & & & & & \\
\hline 2. Clarity & 0.16 & 1 & & & & & & & & \\
\hline 3. Sensations & 0.19 & 0.31 & 1 & & & & & & & \\
\hline 4. Understanding & 0.28 & 0.22 & 0.17 & 1 & & & & & & \\
\hline 5. Acceptance/tolerance & 0.35 & 0.35 & 0.28 & 0.20 & 1 & & & & & \\
\hline 6. Readiness to confront & 0.27 & 0.34 & 0.24 & 0.22 & 0.34 & 1 & & & & \\
\hline 7. Self-support & 0.25 & 0.23 & 0.26 & 0.19 & 0.33 & 0.18 & 1 & & & \\
\hline 8. Modification & 0.18 & 0.34 & 0.34 & 0.22 & 0.36 & 0.32 & 0.18 & 1 & & \\
\hline 9. ERSQ-total & 0.54 & 0.60 & 0.58 & 0.51 & 0.74 & 0.60 & 0.55 & 0.61 & 1 & \\
\hline 10. BSI-total & -0.26 & -0.26 & -0.13 & -0.18 & -0.30 & -0.30 & -0.29 & -0.32 & -0.41 & 1 \\
\hline
\end{tabular}

Note: All correlations were significant at $\mathrm{P}<0.01$.

Iranian Rehabilitation Journal

bance. Moreover, the ability to modify emotions could play complete mediating roles between the variables of attention, bodily perception of sensations, understanding of emotions, and psychological disturbance. However, modification played a partial mediating role in the relationship between emotional clarity, acceptance and tolerance, readiness for confrontation, and self-support.

Several studies have been conducted considering the role and importance of emotion regulation skills on mental health $[11,33]$. Despite the studies in this domain, the superiority of the Berking model (2010) is its comprehensive approach towards emotion regulation skills and their interaction [14]. The results of the present study were similar to the assumptions raised by Berking et al. (2012); nevertheless, the modification ability failed to mediate the relationship between emotional clarity, readiness to confrontation, self-support, and psychopathology in a complete manner. Previous studies showed that the ability to accept, tolerate, and modify the emotions can explain mental health and the outcome of the treatment. Also, the ability to tolerate negative emotions after controlling other emotional emotion skills was predictive of alcohol consumption [12].

Furthermore, the skills of clarity, self-support, modification, and acceptance and tolerance also revealed that this set of skills has been highlighted in the treatment of

Table 3. Effect of emotion regulation skills on psychopathology

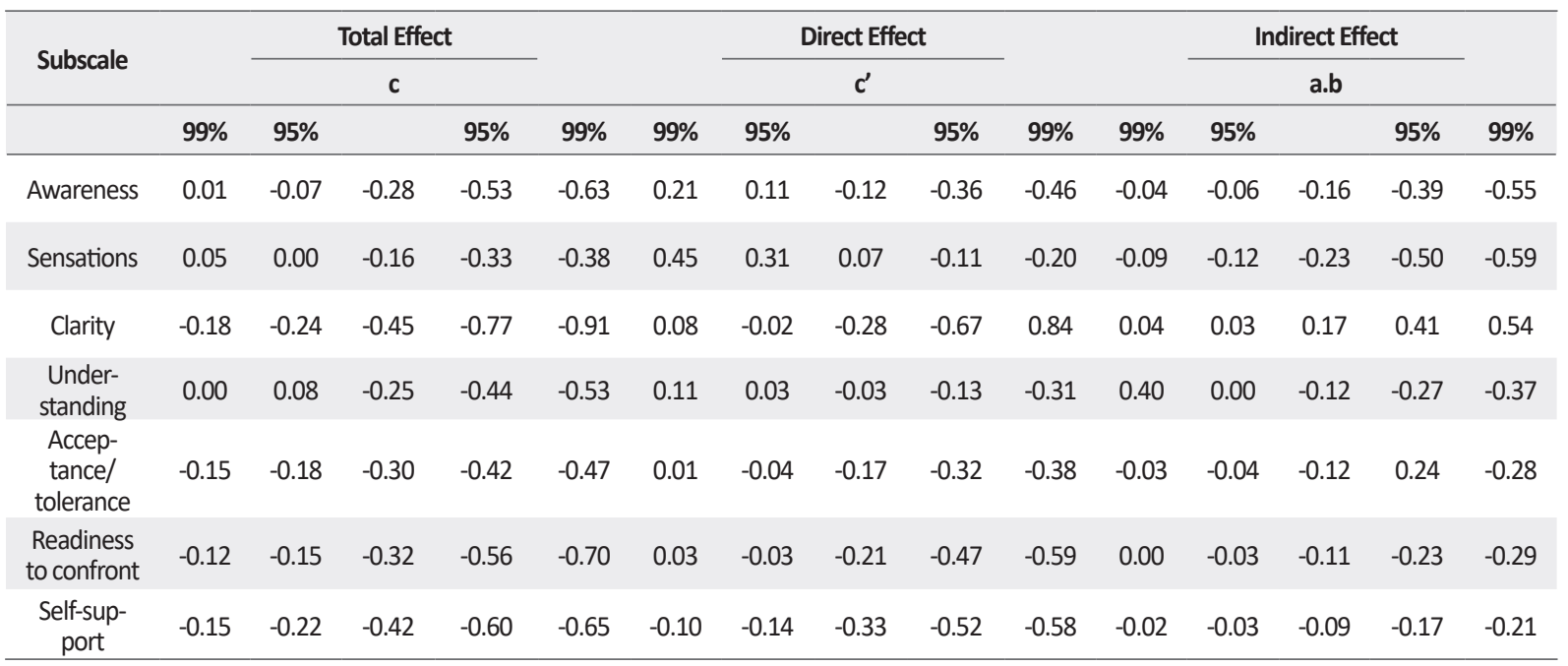




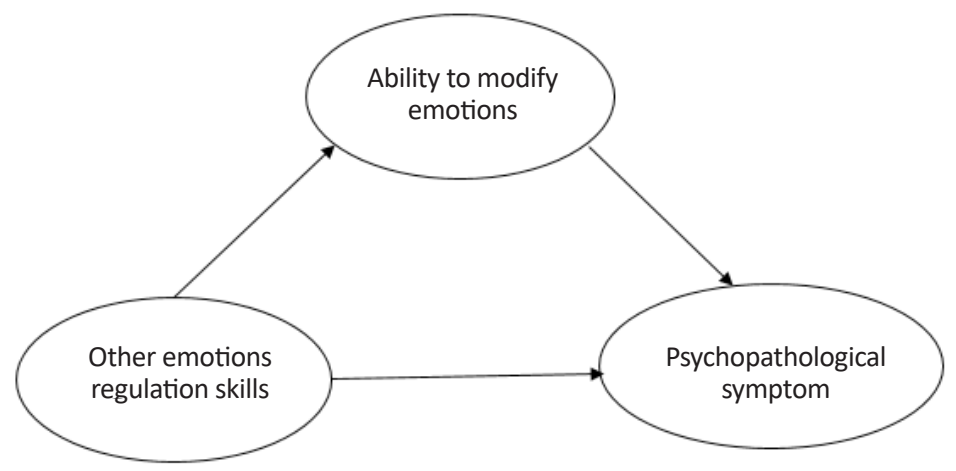

Iranian Rehabilitation Journal

Figure 1. Model with direct and indirect effects for the effect of emotional skills on symptoms (BSI) mediated by modification

the third wave of mindfulness-based therapy, self-compassion therapy, and dialectical behavior therapy. Moreover, the partial mediation of modification between these skills and psychopathology requires that these skills be considered in the treatment plan, altogether. Intriguingly, according to the results of the present study, one of the critical therapeutic considerations indicated by Barking et al. [15] is the goal of psychotherapy in many cases: emotional clarity and non-judgmental awareness of emotions [34]. Nevertheless, the focus of therapy on improving the skills of clarity and non-judgmental awareness is usually not followed by improving the acceptance/tolerance and modification. The non-flexible focus on improving the skills such as attention and clarity of emotions may raise concern. In this process of emotion regulation therapy, patients become aware of emotions beforehand and can recognize and label them accurately. However, if tolerance, acceptance, and modification skills were not considered previously, the individual would experience several emotions that are unacceptable and cannot be modified adaptively [15]. Thus, the therapists need to evaluate the extent of improvements in the skills of acceptance/tolerance and modification simultaneously with improved awareness and emotional clarity. If acceptance/tolerance and modification are not improved, selecting an approach that includes the techniques for direct facilitation of modification and tolerance skills is imperative [15].

The results presented above support one of the most basic principles of dialectical behavioral therapy. According to the overall results of the present study, modification, emotional clarity, acceptance and tolerance, confrontation, and self-support are vital for the mental health. Nonetheless, these skills affected the mental health in two ways. Initially, these skills could show their impact by reducing the severity and the duration of negative emotional states. Then, the acceptance and tolerance of negative emotions were effectuated in short-term if it was not possible to change such states. The change, besides in addition to acceptance, is considered as one of the most basic principles of dialectical behavior therapy [19].

In summary, Berking et al. (2014) speculated that deficits in these skills render difficulty for people to accept, tolerate, and modify their emotions. These individuals consider their emotions as dangerous and always have high levels of epinephrine, norepinephrine, and cortisol. Such high levels of cortisol exert an inhibitory role in the hippocampal and prefrontal region and activation of amygdala. The lack of emotional skills results in a marked inhibition of the cognitive brain regions. Therefore, learning these skills would ultimately improve the emotional disturbance and activation of the cognitive region of the brain and break the vicious cycles [15].

The design of the present study was cross-sectional, that led to several limitations: use of self-reporting measurement scales, enrolment of University students as study participants, and the use of seven models. In addition, this study was carried out with a relatively small sample size; thus, it is recommended to conduct further investigations using large sample size and longitudinal designs. Berking et al. [19] speculated that the use of the ERSQ can potentially evaluate the general coping with emotions and feelings, while it is possible for an anxious person to imply emotion as anxiety and for a depressed person to consider emotions as sorrow and dysphoria. This issue should be considered in future studies as each of these emotional states may have specific emotional regulation deficits.

\section{Conclusion}

This study provided preliminary evidence for the validity of ACE model in Iranian population, such that it could be repeated in clinical samples. However, the ef- 
ficiency of the model is assessed as it might affect the regulation training in the population.

In summary, the results postulated that clarity, ability to modify, acceptance and tolerance, and readiness for confrontation are considered as the four main skills that should be highlighted as high-ranking goals in therapies. Thus, it is essential to review the given skills with respect to the type of emotions in future studies, especially Iranian population. The model with direct and indirect effects for the effect of emotional skills on symptoms (BSI) mediated by modification is indicated in Figure 1.

\section{Ethical Considerations}

\section{Compliance with ethical guidelines}

Ethical issues (including informed consent, plagiarism, misconduct, data fabrication and/or falsification, double publication and/or submission, and redundancy) have been completely observed by the authors.

\section{Funding}

This research did not receive any specific grant from funding agencies in the public, commercial, or not-forprofit sectors.

\section{Conflict of interest}

The authors declared no conflict of interest.

\section{Acknowledgements}

This study was a part of $\mathrm{PhD}$ dissertation in Department of Clinical Psychology, University of Social Welfare and Rehabilitation Sciences, Tehran, Iran. We are grateful to all the students who participated in this study.

\section{References}

[1] Fernandez KC, Jazaieri H, Gross JJ. Emotion regulation: A transdiagnostic perspective on a new RDoC domain. Cognitive Therapy and Research. 2016; 40(3):426-40. [DOI:10.1007/ s10608-016-9772-2] [PMID] [PMCID]

[2] Zbozinek TD, Rose RD, Wolitzky Taylor KB, Sherbourne C, Sullivan G, Stein MB, et al. Diagnostic overlap of generalized anxiety disorder and major depressive disorder in a primary care sample. Depress Anxiety. 2012; 29(12):1065-71. [DOI:10.1002/da.22026] [PMID] [PMCID]
[3] Clark LA, Watson D. Tripartite model of anxiety and depression: psychometric evidence and taxonomic implications. Journal of Abnormal Psychology. 1991; 100(3):316-36. [DOI:10.1037/0021-843X.100.3.316] [PMID]

[4] Barlow DH, Sauer Zavala S, Carl JR, Bullis JR, Ellard KK. The nature, diagnosis, and treatment of neuroticism. Clinical Psychological Science. 2013; 2(3):344-65. [DOI:10.1177/2167702613505532]

[5] McEvoy PM, Watson H, Watkins ER, Nathan P. The relationship between worry, rumination, and comorbidity: Evidence for repetitive negative thinking as a transdiagnostic construct. Journal of Affective Disorders. 2013; 151(1):313-20. [DOI:10.1016/j.jad.2013.06.014] [PMID]

[6] Aldao A. Emotion regulation strategies as transdiagnostic processes: A closer look at the invariance of their form and function. Revista de Psicopatologia y Psicologia Clinica. 2012; 16:261-77. [DOI:10.5944/rppc.vol.17.num.3.2012.11843]

[7] Kring AM, Sloan DM. Emotion regulation and psychopathology: A transdiagnostic approach to etiology and treatment. New York, NY: Guilford Press; 2009.

[8] Insel T, Cuthbert B, Garvey M, Heinssen R, Pine DS, Quinn $\mathrm{K}$, et al. Research Domain Criteria (RDoC): Toward a new classification framework for research on mental disorders. The American Journal of Psychiatry. 2010; 167(7):748-51. [DOI:10.1176/appi.ajp.2010.09091379] [PMID]

[9] Thompson RA. Emotion regulation: A theme in search of definition. Monographs of the Society for Research in Child Development. 2008; 59(2-3):25-52. [DOI: 10.1111/j.15405834.1994.tb01276.x]

[10] Aldao A, Nolen-Hoeksema S, Schweizer S. Emotionregulation strategies across psychopathology: A meta-analytic review. Clinical Psychology Review. 2010; 30(2):217-37. [DOI:10.1016/j.cpr.2009.11.004] [PMID]

[11] Berking M, Wupperman P. Emotion regulation and mental health: recent findings, current challenges, and future directions. Current Opinion in Psychiatry. 2012; 25(2):128-34 [DOI:10.1097/YCO.0b013e3283503669] [PMID]

[12] Berking M, Margraf M, Ebert D, Wupperman P, Hofmann SG, Junghanns K. Deficits in emotion-regulation skills predict alcohol use during and after cognitive-behavioral therapy for alcohol dependence. Journal of Consulting and Clinical Psychology. 2011; 79(3):307-18. [DOI:10.1037/a0023421] [PMID] [PMCID]

[13] Berking M, Orth U, Wupperman P, Meier LL, Caspar F. Prospective effects of emotion-regulation skills on emotional adjustment. Journal of Counseling Psychology. 2008; 55(4):485-94. [DOI:10.1037/a0013589] [PMID]

[14] Berking M. Training emotionaler kompetenzen. Heidelberg: Springer; 2010. [DOI:10.1007/978-3-642-05230-9]

[15] Berking M, Whitley B. Affect regulation training: A practitioners' manual. New York: Springer-Verlag; 2014 [DOI:10.1007/978-1-4939-1022-9]

[16] Berking M, Meier C, Wupperman P. Enhancing emotionregulation skills in police officers: Results of a pilot controlled study. Behavior Therapy. 2010; 41(3):329-39. [DOI:10.1016/j. beth.2009.08.001] [PMID] 
[17] Berking M, Wupperman P, Reichardt A, Pejic T, Dippel A, Znoj H. Emotion-regulation skills as a treatment target in psychotherapy. Behaviour Research and Therapy. 2008; 46(11):1230-7. [DOI:10.1016/j.brat.2008.08.005] [PMID]

[18] Berking M, Znoj H. [Development and validation of a selfreport measure for the assessment of emotion-regulation skills (German)]. Zeitschrift für Psychiatrie, Psychologie und Psychotherapie. 2008; 56(2):141-53. [DOI:10.1024/16614747.56.2.141]

[19] Berking M, Poppe C, Luhmann M, Wupperman P, Jaggi $V$, Seifritz E. Is the association between various emotionregulation skills and mental health mediated by the ability to modify emotions? Results from two cross-sectional studies. Journal of Behavior Therapy and Experimental Psychiatry. 2012; 43(3):931-7. [DOI:10.1016/j.jbtep.2011.09.009] [PMID]

[20] Hayes SC. Acceptance and commitment therapy, relational frame theory, and the third wave of behavioral and cognitive therapies. Behavior Therapy. 2004; 35(4):639-65. [DOI:10.1016/S0005-7894(04)80013-3]

[21] Ford BQ, Mauss IB. Culture and emotion regulation. Current Opinion in Psychology. 2015; 3:1-5. [DOI:10.1016/j.copsyc.2014.12.004]

[22] Soto JA, Perez CR, Kim YH, Lee EA, Minnick MR. Is expressive suppression always associated with poorer psychological functioning? A cross-cultural comparison between European Americans and Hong Kong Chinese. Emotion (Washington, DC). 2011; 11(6):1450-5. [DOI:10.1037/a0023340] [PMID]

[23] Matsumoto D, Yoo SH, Nakagawa S. Culture, emotion regulation, and adjustment. Journal of personality and social psychology. 2008; 94(6):925-37. [DOI:10.1037/00223514.94.6.925] [PMID]

[24] English T, John OP. Understanding the social effects of emotion regulation: the mediating role of authenticity for individual differences in suppression. Emotion (Washington, DC). 2013; 13(2):314-29. [DOI:10.1037/a0029847] [PMID]

[25] Wei M, Su JC, Carrera S, Lin SP, Yi F. Suppression and interpersonal harmony: A cross-cultural comparison between Chinese and European Americans. Journal of Counseling Psychology. 2013; 60(4):625-33. [DOI:10.1037/a0033413] [PMID]

[26] Su JC, Wei M, Tsai HT. Running away from unwanted feelings: culture matters. Cognition \& Emotion. 2014; 28(7):1313 27. [DOI:10.1080/02699931.2014.881322] [PMID]

[27] Mohammadi R, Bahari Z, Robat MS, SMR SA, Karimi R. [Reliability, validity, and normalization of Berking's Emotion- Regulation Skills Questionnaire in the students of Police University (Persian)]. Journal of Police Medicine. 2015; 4(2):105-112.

[28] Derogaitis L. Manual of brief symptom inventory, administration, scoring and procedure manual. New York: Willy Press; 2000.

[29] Stefan Dabson K, Mohammadkhani P, Massah Choulabi O. Psychometrics characteristic of Beck Depression Inventory-II in patients with magor depressive disorder. Archives of Rehabilitation. 2007; 8:82.

[30] Mohammadkhani P, Dobson KS, Amiri M, Hosseini Ghafari F. Psychometric properties of the Brief Symptom Inventory in a sample of recovered Iranian depressed patients.
International Journal of Clinical Health \& Psychology. 2010; 10(3):50-72

[31] Baron RM, Kenny DA. The moderator-mediator variable distinction in social psychological research: Conceptual, strategic, and statistical considerations. Journal of Personality and Social Psychology. 1986; 51(6):1173-82. [DOI:10.1037/00223514.51.6.1173] [PMID]

[32] Sobel ME. Asymptotic confidence intervals for indirect effects in structural equation models. Sociological methodology. 1982; 13:290-312. [DOI:10.2307/270723]

[33] Hu T, Zhang D, Wang J, Mistry R, Ran G, Wang X. Relation between emotion regulation and mental health: A metaanalysis review. Psychological Reports. 2014; 114(2):341-62. [DOI:10.2466/03.20.PR0.114k22w4] [PMID]

[34] Kuyken W, Watkins E, Holden E, White K, Taylor RS, Byford S, et al. How does mindfulness-based cognitive therapy work. Behaviour Research and Therapy. 2010; 48(11):1105-12. [DOI:10.1016/j.brat.2010.08.003] [PMID] 